\title{
Lacrimal Gland Abscess leading to Frontal Bone Chronic Osteomyelitis: A Rare Case Report
}

\author{
${ }^{1}$ Saurabh Varshney, ${ }^{2}$ Manu Malhotra, ${ }^{3}$ Navjot Kaur, ${ }^{4}$ Priyanka Gairola
}

\begin{abstract}
We report a case of a 15-year-old girl presenting with left lacrimal abscess with chronic osteomyelitis of frontal bone, a rare sequelae, that of lacrimal adenitis. If lacrimal adenitis is left untreated, abscess formation can occur and can also lead to osteomyelitis. If lacrimal adenitis/abscess is diagnosed early in the disease process by understanding typical signs, conservative management can accomplish resolution without the complications and need for surgical exploration and the cosmetic implications of facial surgery.
\end{abstract}

Keywords: Frontal bone, Lacrimal abscess, Osteomyelitis.

How to cite this article: Varshney S, Malhotra M, Kaur N, Gairola P. Lacrimal Gland Abscess leading to Frontal Bone Chronic Osteomyelitis: A Rare Case Report. Clin Rhinol An Int J 2016;9(2):102-104.

\section{Source of support: Nil}

Conflict of interest: None

\section{INTRODUCTION}

Dacryoadenitis is an acute or chronic inflammation of lacrimal gland caused by a variety of microorganisms and can lead to abscess formation. Infections may involve lacrimal gland secondarily by hematogenous route or by microorganisms traveling through ductules. It can be of noninfective variety too. Lacrimal gland abscesses are potentially dangerous infections of orbit and it is a rare clinical entity. ${ }^{1}$

\section{CASE REPORT}

A 15-year-old female presented with recurrent intermittent swelling over the left eyelid for the last 6 months. She was prescribed oral antibiotics and topical antibiotic eye drops by a local practitioner, but to no relief and swelling used to reappear again after an interval of time. Swelling was accompanied by redness in the same eye with mild frontal

\footnotetext{
${ }^{1}$ Professor and Head, ${ }^{2}$ Assistant Professor, ${ }^{3}$ Ex Senior Resident ${ }^{4}$ Senior Resident

${ }^{1-4}$ Department of Otorhinolaryngology and Head and Neck Surgery, All India Institute of Medical Sciences, Rishikesh Uttarakhand, India

Corresponding Author: Saurabh Varshney, Professor and Head, Department of Otorhinolaryngology and Head and Neck Surgery, All India Institute of Medical Sciences, Rishikesh Uttarakhand, India, e-mail: drsaurabh68@gmail.com
}

headache, but there was no history of discharge from eyes or nose, difficulty of vision, and there was no history of trauma or insect bite. There was no history suggestive of any systemic or chronic disease.

Local examination revealed a $6 \times 3 \mathrm{~cm}$ swelling over left upper eyelid and left eyebrow. On palpation, it was soft, cystic, tender, fluctuant, and nonpulsatile. Temperature over the swelling was raised and reddish discoloration of the overlying skin was seen. Rest of the eye findings were normal. There was no change in visual acuity, and ocular movements were intact except slight restriction in lateral movements in the same eye. There was no proptosis. Routine ENT and head-neck examination was normal (Figs 1 and 2).

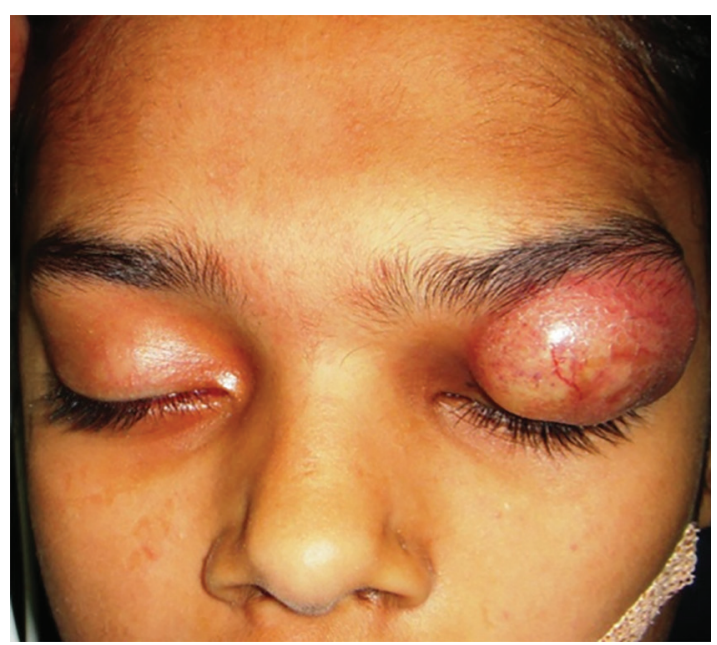

Fig. 1: Left lacrimal abscess

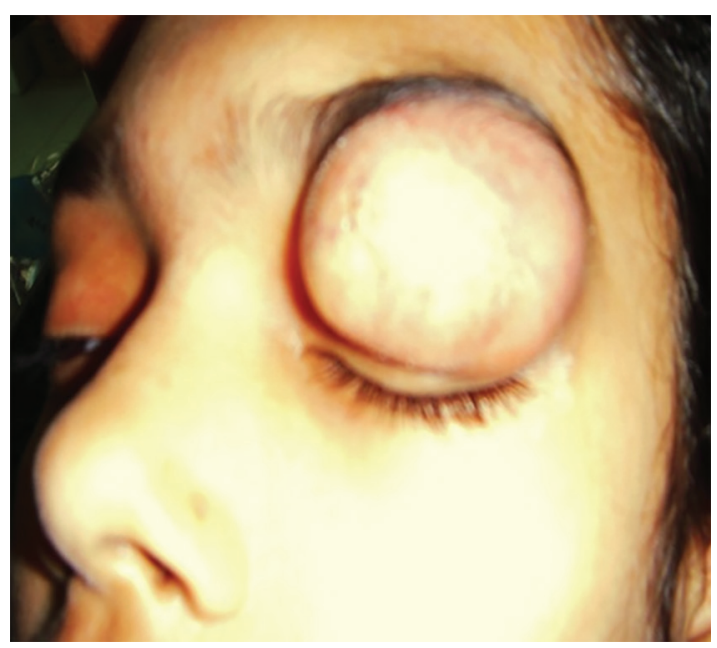

Fig. 2: Left lacrimal abscess 


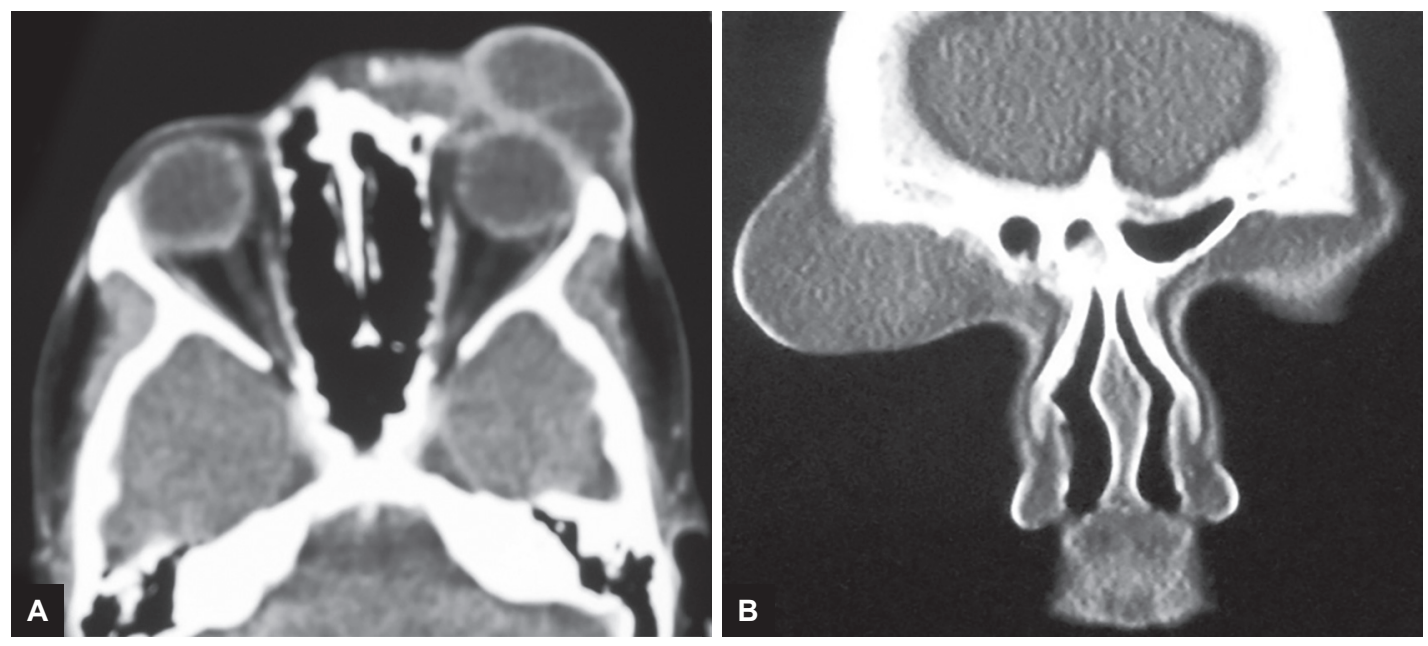

Figs $3 A$ and B: Axial and coronal section of MRI, showing a peripherally enhancing collection anterior to the frontal bone on left side extending up to the anterior aspect of left eyelid

Body temperature was $38^{\circ} \mathrm{C}$ and there were no signs of any systemic disease or adjacent skin infection. Hematological parameters were within normal limits except raised ESR. Serum angiotensin-converting enzyme (ACE) levels were normal, ruling out sarcoidosis. Conjunctival swab did not grow any organism.

Magnetic resonance imaging (MRI) features of nose, paranasal sinuses, and orbit were suggestive of a peripherally enhancing collection anterior to the frontal bone on left side extending up to the anterior aspect of left eyelid (Figs 3A and B).

Incision and drainage was planned. Horizontal incision as extended Lynch-Howarth incision was given over the swelling. Pus was drained and sent for gram staining, acid-fast bacilli (AFB), and culture sensitivity. Tissue was dissected and biopsy taken from lacrimal gland region, which was later sent for histopathological examination and for mycobacterium tuberculosis polymerase chain reaction (MTB-PCR). No pyogenic organism grew on culture and AFB staining came out

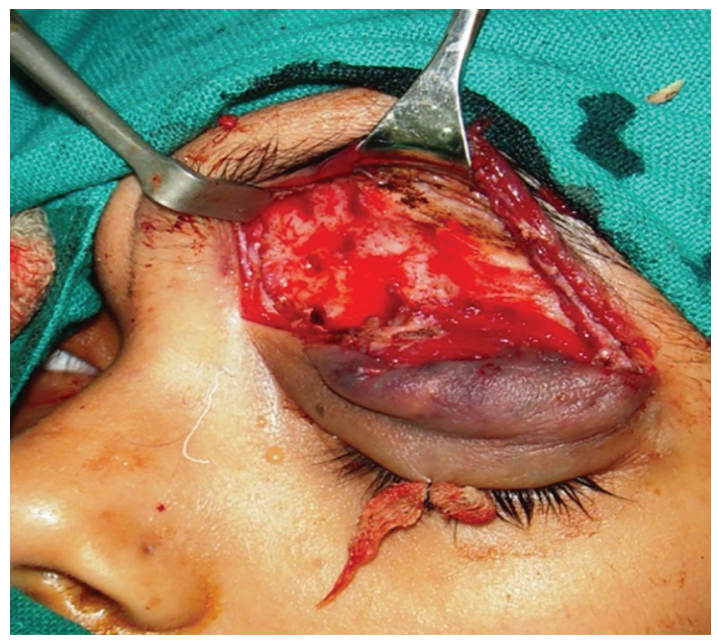

Fig. 4: Intraoperative photograhs to be negative. Intraoperative findings revealed necrotic frontal bone due to chronic osteomyelitis for which sequestrectomy was done (Figs 4 and 5).

Histopathological examination (HPE) showed nonnecrotizing granulomatous inflammation with separate necrosis, foamy histiocytes, hemosiderin laden macrophages, and superadded acute inflammation. There was no surviving lacrimal gland tissue. The MTB-PCR results came out to be negative.

A diagnosis of lacrimal gland abscess leading to chronic osteomyelitis of frontal bone was made. Postoperatively, broad-spectrum antibiotics were given. Stitches were removed on postoperative day 7. Postoperative recovery was satisfactory and she became asymptomatic after 2 weeks (Fig. 6). Postoperative computed tomography (CT) was normal after 3 months (Fig. 7).

\section{DISCUSSION}

Dacryoadenitis is an unusual orbital infection that rarely involves acute suppuration. Infectious causes of

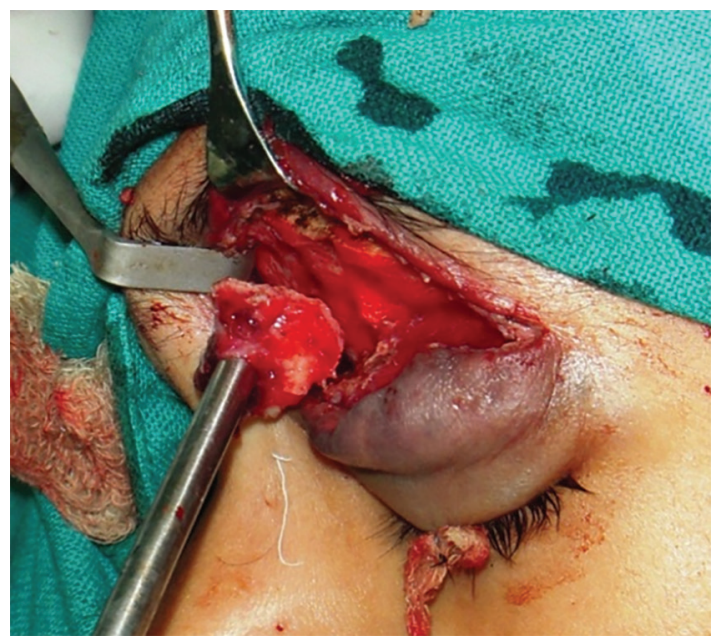

Fig. 5: Intraoperative photograhs 


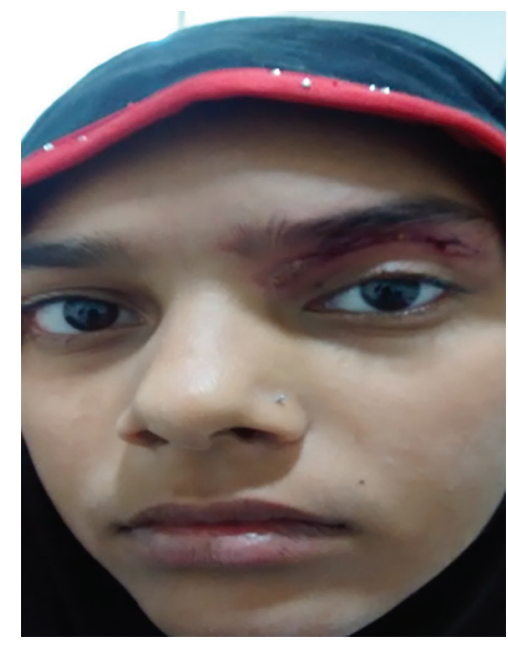

Fig. 6: Postoperative day 14

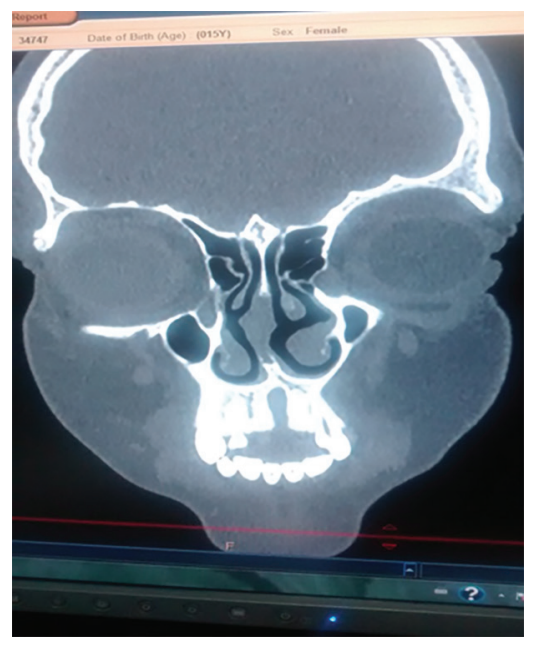

Fig. 7: Postoperative CT (after 3 months) the lacrimal gland may be secondary to hematogenous seeding of the gland or by microorganisms ascending through the ductules. Causes of infectious dacryoadenitis include viruses, bacteria, fungi, and parasites. It may be noninfective too. ${ }^{2}$ Very few cases have been reported of lacrimal gland abscess ${ }^{3}$ and ours is one such case with idiopathic etiology.

Infectious diseases of lacrimal gland usually develop as a complication of sinusitis or due to obstructed lacrimal gland drainage. ${ }^{4-7}$

Acute dacryoadenitis is an infection of the lacrimal gland characterized by pain, tenderness, and swelling of the lacrimal gland. Localized abscess of the lacrimal gland may be seen. ${ }^{2}$ Acute dacryoadenitis may result from systemic infections like measles, mumps, influenza, and may occur subsequent to staphylococcal conjunctivitis. ${ }^{8-10}$ Noninfective causes, such as pseudotumor can give rise to dacryoadenitis. ${ }^{11,12}$

Treatment of acute suppurativa dacryoadenitis is systemic and topical antibiotics. Although rare, lacrimal gland abscess may still occur and may require surgical drainage if spontaneous resolution does not occur. ${ }^{3}$

In our case, frontal bone osteomyelitis was seen secondary to lacrimal gland abscess which was confirmed intraoperatively. As osteomyelitis of skull itself is a rare clinical presentation, it usually occurs by direct extension of infection from paranasal sinuses or by retrograde thrombophlebitis. Its intracranial complications can be life-threatening, therefore early diagnosis and appropriate management becomes essential. ${ }^{13}$

Lacrimal abscess is usually a complication of acute sinusitis but in our case, there were no signs and symptoms of sinusitis, and on histopathology examination evidence of malignancy was ruled out. Earlier osteomyelitis was suspected by history and clinical presentation which was confirmed intraoperatively. So this case was of idiopathic acute suppurative dacryoadenitis complicated by lacrimal abscess formation leading to chronic osteomyelitis of frontal bone.

A diagnosis of lacrimal gland abscess should always be kept in mind in a case of eyelid swelling with pain and tenderness. Due to rarity of lacrimal gland abscess, index of suspicion is low, therefore this case is presented.

\section{REFERENCES}

1. Chandravanshi SL, Mishra V. Acute suppurative bacterial dacryoadenitis: a rare case report. Eur J Ophthalmol 2014 Sep-Oct;24(5):790-792.

2. Tabbara KF, Abu-El-Asrar AM, Khairallah M. Ocular infections. 1st ed. Berlin, Heidelberg: Springer; 2014. p. 48.

3. McCabe AA. Lacrimal gland abscess: two case reports. Aust NZ J Ophthalmol 1999 Feb;27(1):75-78.

4. Owens D, Olima B, Winter R, William H. Lacrimal cellulitis: an unusual complication of acute sinusitis. Internet J Otorhinolaryngol 2006;6(2).

5. Chandeler JR, Langenbrunner DJ, Stevens ER. The Pathogenesis of orbital complications in acute sinusitis. Laryngoscope 1970 Sep;80(9):1414-1428.

6. Harris GJ, Synder RW. Lacrimal gland abscess. Am J Ophthalmol 1987 Aug;104(2):193-194.

7. Mizra S, Lobo C, Counter P, Farrington WT. Lacrimal gland abscess: an unusual complication of rhinosinusitis. ORL J Otolaryngol Relat Spec 2001 Nov-Dec;63(6):379-381.

8. Duke Elder S. Diseases of the outer eye, cornea and sclera. In: Duke Elder S, editor. System of ophthalmology. St. Louis (MO): Mosby; 1965. p. 991-992.

9. Madhkar K, Bhide M, Prasad CE, Venkatranayya. Tuberculosis of the lacrimal gland. J Trop Med Hyg 1991 Jun;94(3):150-151.

10. Malthieu D, Turut P, Francois Y. Dacryoadenitis and sarcoidosis. Bull Soc Ophthalmol Fr 1985 Jan;85(1):65-66.

11. Kennerdell JS, Dresner SC. The non-specific orbital inflammatory syndromes. Surv Ophthalmol 1984 Sep-Oct;29(2):93-103.

12. Sekhar GC, Mandal AK, Vyas P. Non-specific orbital inflammatory diseases. Doc Ophthalmol 1993;84(2):155-170.

13. Osei-Yeboah C, Neequaye J, Bulley H, Darkwa A. Osteomyelitis of the frontal bone. Ghana Med J 2007 Jun;41(2): $88-90$. 